\title{
A vigilância epidemiológica no âmbito municipal: avaliação do grau de implantação das ações
}

\author{
Epidemiological surveillance at the municipal level: \\ evaluation of the degree of implementation
}

\author{
${ }^{1}$ Centro de Pesquisas Aggeu \\ Magalhães, Fundação \\ Oswaldo Cruz, Recife, Brasil. \\ 2 Instituto de Medicina \\ Integral Professor Fernando \\ Figueira, Recife, Brasil. \\ Correspondência \\ L. C. A. Bezerra \\ Departamento de Saúde \\ Coletiva, Centro de Pesquisas \\ Aggeu Magalhães, Fundação \\ Oswaldo Cruz. \\ Rua Ribeiro Pessoa 324/B8, \\ Recife, $P E$ \\ 50880-580, Brasil. \\ lua_cad@yahoo.com.br \\ lucianacaroline@imip.org.br
}

\section{Abstract}

This study's objective was to evaluate the degree of implementation of epidemiological surveillance activities by the Municipal Health Secretariat in Recife, Pernambuco State, Brazil. The design consisted of a case study with a normative approach. The logical model for epidemiological surveillance was outlined as the basis for selecting criteria that comprised a matrix submitted to specialists using the consensus technique or Delphi Method. This consensual matrix was used as the basis for the questionnaires used in the interviews. Three analytical levels were approached: the central level, 6 health districts, and 62 family health teams. According to the study, epidemiological surveillance has been partially implemented in the municipality, based on the degree of implementation at the three levels (already implemented at the central level and partially implemented in the health districts and family health teams). Despite the decentralization of epidemiological surveillance activities to the municipalities, different levels in the municipal system have different needs in dealing with the standards and norms, including the elaboration of internal guidelines to define responsibilities at the three levels.

Health Evaluation; Family Health; Epidemiologic Surveillance
Luciana Caroline Albuquerque Bezerra 1,2

Eduardo Freese 1

Paulo Germano de Frias 2

Isabella Samico 2

Cínthia Kalyne Alves de Almeida 2

\section{Introdução}

A descentralização das ações e serviços do Sistema Único de Saúde (SUS) tem contribuído para a qualificação do sistema de saúde, na medida em que o aproxima das especificidades epidemiológicas, sociais, políticas e administrativas de cada local, buscando superar as fragmentações das políticas e programas por meio de uma rede hierarquizada e regionalizada de ações 1 .

No sentido de reorganizar o funcionamento do sistema de saúde, em 2006, é proposto o Pacto pela Saúde. O pacto sugere mudanças significativas na relação entre os três níveis de gestão, dando ênfase a uma atitude dialógica tanto na definição das prioridades sanitárias quanto na gestão do SUS, e prevê o financiamento federal para estados e municípios por blocos de recursos. A vigilância à saúde constitui um desses blocos, tendo como um dos componentes a vigilância epidemiológica com recursos destinados às ações de vigilância, prevenção e controle de doenças. A formalização desse pacto se dá por intermédio de um termo de compromisso de gestão que contém as atribuições de cada gestor, dentre elas que todo município deve assumir a gestão e execução das ações de vigilância em saúde, compreendendo as ações de vigilância epidemiológica, sanitária e ambiental, de acordo com as normas vigentes e pactuações estabelecidas 1 . Todavia, ainda não se vislumbram as implicações do pacto pela saúde no SUS, visto que ainda é uma 
possibilidade 2 . A efetivação dos acordos entre as três esferas e a construção de processos e instrumentos que reconheçam as diversidades locais constituem desafios concretos do pacto ${ }^{3}$.

Antes disso, algumas normas e pactuações foram instituídas e estão sendo implementadas como estratégias para promover a transformação do modelo vigente. O Guia de Vigilância Epidemiológica 4; a Programação Pactuada e Integrada (PPI-VS) 5 e o projeto de reestruturação da vigilância epidemiológica (o VIGISUS) 6 conformam tentativas de modificar e viabilizar a prática da vigilância nos diversos níveis de gestão, qualificando as ações desenvolvidas e as informações produzidas.

Nesse sentido, o uso efetivo e sistemático das informações da vigilância epidemiológica torna-se importante ferramenta tanto na gestão do sistema de saúde para definição das prioridades e distribuição de recursos quanto para nortear as estratégias de intervenção dos serviços de saúde 4 . Entretanto, dificuldades estruturais do sistema de saúde 7 e da própria integração das ações de vigilância com as ações assistenciais nos serviços 8 têm demonstrado um caráter meramente descritivo, restrito à coleta de dados, e por vezes, à transmissão destes dados a outros níveis 4.

Apreendendo-se a complexidade do desenvolvimento das ações de vigilância epidemiológica, uma vez que estas são realizadas em níveis distintos do sistema de saúde, torna-se imprescindível saber se os municípios têm conseguido responder às demandas da descentralização. Iniciativas institucionais e pesquisas têm sido realizadas, no intuito de avaliar a atuação da vigilância epidemiológica em diversos âmbitos do sistema de saúde 7,8,9,10, o que sugere a necessidade da permanente avaliação das ações de vigilância epidemiológica.

Considerando a importância da avaliação das práticas de saúde como um instrumento norteador dos serviços prestados, este estudo tem o objetivo de avaliar o grau de implantação da vigilância epidemiológica no âmbito municipal, de modo a contribuir para a tomada de decisão.

\section{Método}

A estratégia adotada foi o estudo de caso, utilizando-se uma abordagem normativa da vigilância epidemiológica. O caso, a vigilância epidemiológica da Secretaria Municipal de Saúde do Recife, Estado de Pernambuco, foi analisado a partir do delineamento do modelo lógico da vigilância epidemiológica. Esse foi elaborado com base em uma análise documental utilizando- se informações contidas no Guia de Vigilância Epidemiológica da Fundação Nacional de Saúde (2005), na PPI-VS (2006), no Pacto de Atenção Básica (2006), em normas/portarias que regulamentam as ações de vigilância epidemiológica, além dos relatórios da Gerência Municipal de Vigilância Epidemiológica.

O modelo lógico da vigilância epidemiológica no âmbito municipal explicita dois componentes: Gestão da Vigilância Epidemiológica, com seis subcomponentes (Recursos Humanos, Normatização, Planejamento, Monitoramento/ Avaliação, Integração das Ações, Informação) e Desenvolvimento das Ações de Vigilância Epidemiológica com cinco subcomponentes (Notificação, Diagnóstico, Busca Ativa, Investigação e Educação em Saúde). Para cada componente consideraram-se os insumos, as atividades e os resultados esperados, como podem ser vistos na Figura 1.

Baseando-se no modelo lógico foi construída uma matriz de julgamento, na qual foram elencados critérios/indicadores para cada subcomponente (Tabela 1). Para garantir a validade de conteúdo e de construção do modelo lógico e da matriz de julgamento foi realizada uma técnica de consenso (método Delphos) com nove especialistas, segundo a metodologia descrita por Souza et al. 11. Com base na matriz consensuada pelos especialistas foram elaborados os questionários das entrevistas.

Precederam o trabalho de campo: o piloto com $10 \%$ da amostra (seis equipes) para validação e ajustes dos questionários; a capacitação e o treinamento específico dos entrevistadores para a aplicação do instrumento e consolidação das respostas.

Considerou-se três níveis de análise: nível central, distritos sanitários e equipes de saúde da família. A coleta de dados foi realizada em novembro de 2005 e utilizou entrevistas semi-estruturadas. Os respondentes foram os coordenadores da vigilância epidemiológica municipal (1), distritais (6) e médicos ou enfermeiros das equipes de saúde da família (62). O critério utilizado para seleção dos informantes-chave foi o fato de serem os responsáveis pelas ações de vigilância epidemiológica nesses níveis e os que detinham maior conhecimento das atividades realizadas no âmbito de sua atuação.

Para as equipes de saúde da família definiuse arbitrariamente uma amostra aleatória de 1/3 de equipes existentes no município em novembro de 2005, totalizando 62 . A opção se sustenta na estratégia da pesquisa ao adotar o estudo de caso, que considera na validade interna a consistência, coerência e qualidade da articulação teórica do modelo, e na validade externa privilegiam 


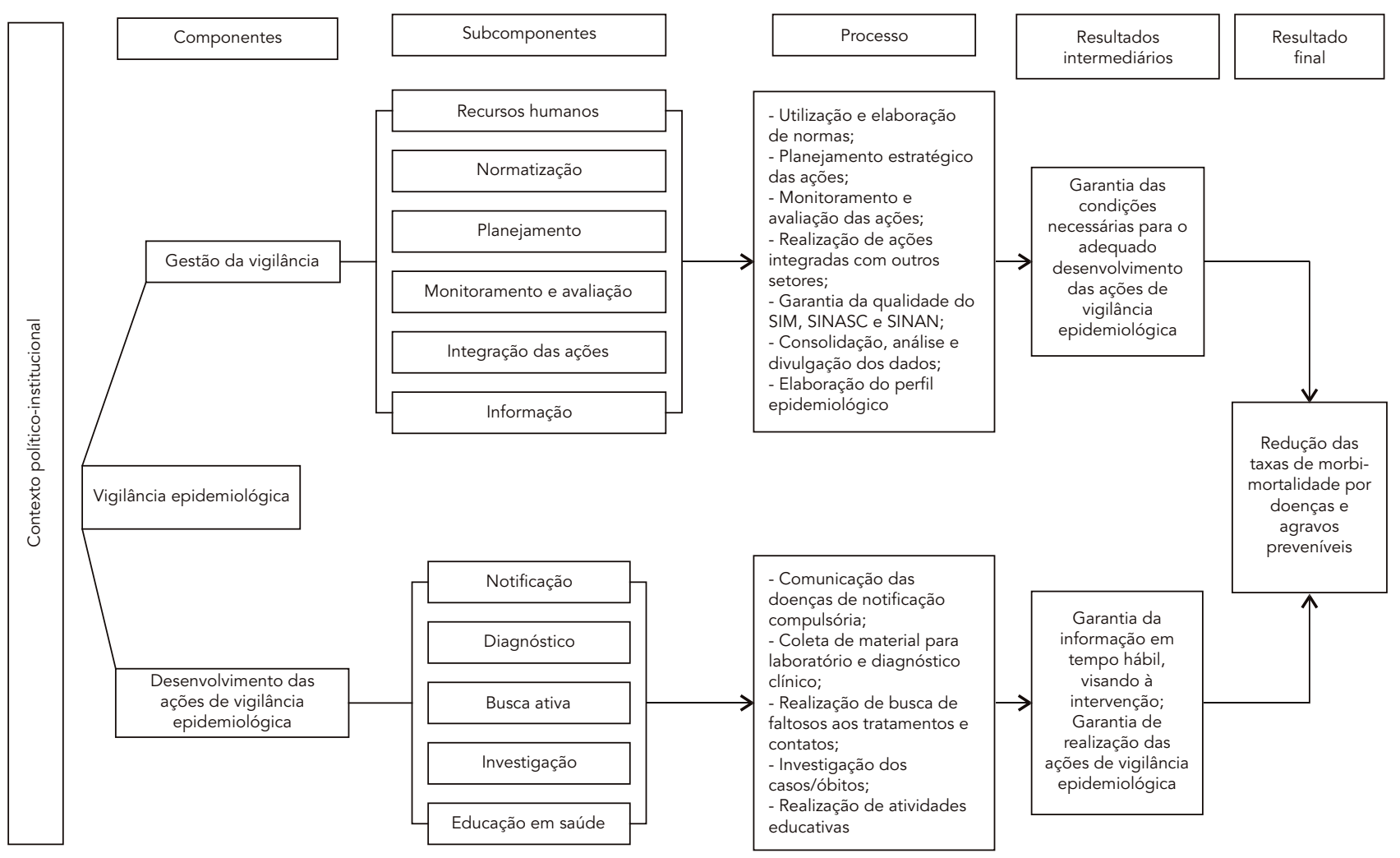

SIM: Sistema de Informações sobre Mortalidade; SINASC: Sistema de Informações de Nascidos Vivos; SINAN: Sistema de Informação de Agravos de Notificação.

a generalização analítica mais que a estatística ao tomar como referência o modelo adotado para ser confrontado com a realidade empírica.

Em algumas situações para a apreensão da realidade empírica e operacionalização da avaliação do componente utilizaram-se os conceitos de condição traçadora 12 e de evento sentinela 13 . As condições traçadoras da vigilância epidemiológica municipal adotadas foram: doenças exantemáticas; dengue; tuberculose e hanseníase; e os eventos-sentinela: mortalidade materna e mortalidade infantil.

Os resultados encontrados foram confrontados com o modelo lógico, e o grau de implantação definido a partir da adesão ao preconizado expresso no modelo.

O grau de implantação dos componentes foi obtido pela média aritmética das pontuações dos subcomponentes, aos quais foram atribuídos 10 pontos, divididos entre os critérios de cada subcomponente (Tabela 1). A média aritmética do grau de implantação dos dois componentes resultou no grau de implantação da dimensão unitária da vigilância epidemiológica em cada nível de análise (equipes de saúde da família, distrito sanitário e nível central). O grau de implantação da vigilância epidemiológica no município foi obtido pela média aritmética da dimensão unitária da vigilância epidemiológica em cada nível de análise.

Os pontos de corte adotados para definir o grau de implantação da vigilância epidemiológica foram: implantada para os valores de 8,0 a 10,0; parcialmente implantada para os de 6,0 a 7,9; e não implantada, menor ou igual a 5,9. O sistema de escores e os pontos de corte empregados foram adaptados de Felisberto et al. 14 e de Samico et al. 15, com base, também, nos resultados da conferência de consenso. 
Tabela 1

Matriz de julgamento consensuada pelos especialistas, por componentes e subcomponente do modelo lógico da vigilância epidemiológica. Recife, Pernambuco, Brasil, 2005.

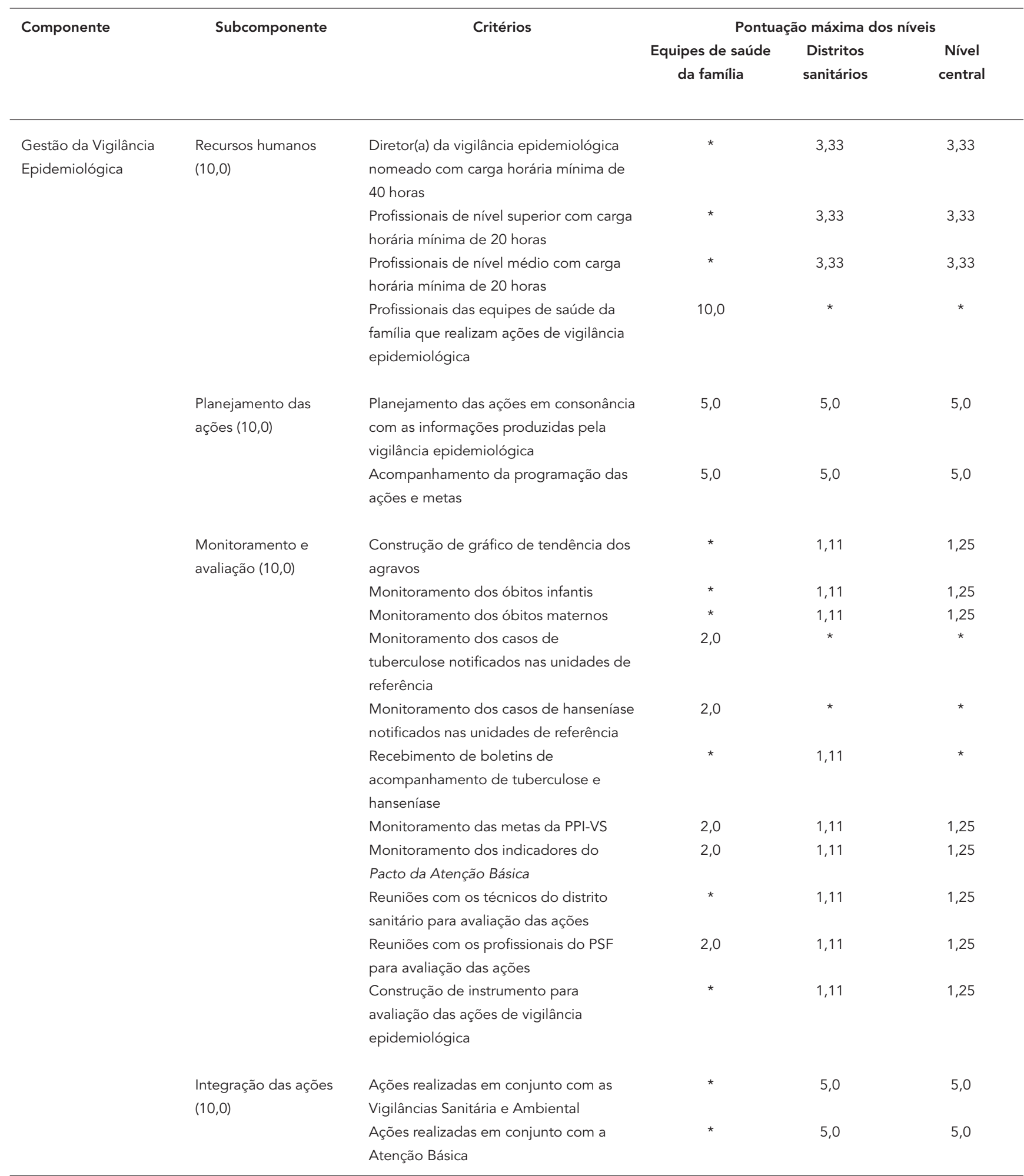

(continua) 
Tabela 1 (continuação)

\begin{tabular}{|c|c|c|c|c|c|}
\hline \multirow[t]{2}{*}{ Componente } & \multirow[t]{2}{*}{ Subcomponente } & \multirow[t]{2}{*}{ Critérios } & \multicolumn{3}{|c|}{ Pontuação máxima dos níveis } \\
\hline & & & $\begin{array}{l}\text { Equipes de saúde } \\
\text { da família }\end{array}$ & $\begin{array}{l}\text { Distritos } \\
\text { sanitários }\end{array}$ & $\begin{array}{l}\text { Nível } \\
\text { central }\end{array}$ \\
\hline \multirow[t]{16}{*}{$\begin{array}{l}\text { Gestão da Vigilância } \\
\text { Epidemiológica }\end{array}$} & Informação $(10,0)$ & $\begin{array}{l}\text { Processamento e análise dos dados no } \\
\text { SIM }\end{array}$ & * & * & 1,0 \\
\hline & & $\begin{array}{l}\text { Processamento e análise dos dados no } \\
\text { SINASC }\end{array}$ & * & * & 1,0 \\
\hline & & $\begin{array}{l}\text { Processamento e análise dos dados no } \\
\text { SINAN }\end{array}$ & * & 1,43 & * \\
\hline & & $\begin{array}{l}\text { Envio do banco de dados do SIM, } \\
\text { SINASC e SINAN para o estado }\end{array}$ & * & * & 1,0 \\
\hline & & $\begin{array}{l}\text { Envio de boletins epidemiológicos aos } \\
\text { outros níveis }\end{array}$ & * & 1,43 & 1,0 \\
\hline & & $\begin{array}{l}\text { Envio dos bancos de dados dos SIS aos } \\
\text { níveis normatizados }\end{array}$ & 2,5 & 1,43 & 1,0 \\
\hline & & $\begin{array}{l}\text { Consolidação e análise dos dados de } \\
\text { vigilância epidemiológica produzidos } \\
\text { pelas equipes de saúde da família }\end{array}$ & 2,5 & * & * \\
\hline & & $\begin{array}{l}\text { Produção de relatórios com a situação } \\
\text { epidemiológica da área }\end{array}$ & 2,5 & * & 1,0 \\
\hline & & Existência de computadores & 2,5 & 1,43 & 1,0 \\
\hline & & Existência de impressoras & * & 1,43 & 1,0 \\
\hline & & Existência de telefones exclusivos & * & 1,43 & 1,0 \\
\hline & & $\begin{array}{l}\text { Suprimento de papel, tinta de } \\
\text { impressora, disquete }\end{array}$ & * & 1,43 & 1,0 \\
\hline & Normatização $(10,0)$ & $\begin{array}{l}\text { Elaboração de normas internas definindo } \\
\text { atribuições de cada nível do sistema }\end{array}$ & * & * & 2,5 \\
\hline & & $\begin{array}{l}\text { Utilização das normas existentes para } \\
\text { realização das ações de vigilância } \\
\text { epidemiológica }\end{array}$ & 3,34 & 3,34 & 2,5 \\
\hline & & $\begin{array}{l}\text { Existência de Leis/Portarias; guias do } \\
\text { Ministério da Saúde }\end{array}$ & 3,33 & 3,33 & 2,5 \\
\hline & & $\begin{array}{l}\text { Existência de normatização interna sobre } \\
\text { as atribuições de cada nível do sistema }\end{array}$ & 3,33 & 3,33 & 2,5 \\
\hline $\begin{array}{l}\text { Desenvolvimento das } \\
\text { Ações de Vigilância }\end{array}$ & Notificação $(10,0)$ & $\begin{array}{l}\text { Notificação dos casos suspeitos de } \\
\text { doenças exantemáticas }\end{array}$ & 1,67 & 2,0 & * \\
\hline \multirow[t]{9}{*}{ Epidemiológica } & & Notificação negativa de sarampo & 1,66 & * & * \\
\hline & & $\begin{array}{l}\text { Notificação de casos suspeitos de } \\
\text { dengue }\end{array}$ & 1,67 & 2,0 & * \\
\hline & & Notificação de casos de tuberculose & 1,67 & 2,0 & * \\
\hline & & Notificação de casos de hanseníase & 1,67 & 2,0 & * \\
\hline & & Existência de fichas de notificação & 1,66 & 2,0 & * \\
\hline & Diagnóstico $(10,0)$ & $\begin{array}{l}\text { Sorologia para diagnóstico de casos de } \\
\text { sarampo }\end{array}$ & 2,5 & 5,0 & * \\
\hline & & $\begin{array}{l}\text { Sorologia para diagnóstico de casos de } \\
\text { dengue }\end{array}$ & 2,5 & $\begin{array}{l}5,0 \\
*\end{array}$ & * \\
\hline & & $\begin{array}{l}\text { Exame clínico e baciloscópico de } \\
\text { suspeitos de hanseníase }\end{array}$ & 2,5 & * & * \\
\hline & & $\begin{array}{l}\text { Baciloscopia direta do escarro de casos } \\
\text { suspeitos de tuberculose }\end{array}$ & 2,5 & 2,5 & * \\
\hline
\end{tabular}

(continua) 
Tabela 1 (continuação)

\begin{tabular}{|c|c|c|c|c|c|}
\hline \multirow[t]{2}{*}{ Componente } & \multirow[t]{2}{*}{ Subcomponente } & \multirow[t]{2}{*}{ Critérios } & \multicolumn{3}{|c|}{ Pontuação máxima dos níveis } \\
\hline & & & $\begin{array}{l}\text { Equipes de saúde } \\
\text { da família }\end{array}$ & $\begin{array}{l}\text { Distritos } \\
\text { sanitários }\end{array}$ & $\begin{array}{l}\text { Nível } \\
\text { central }\end{array}$ \\
\hline $\begin{array}{l}\text { Desenvolvimento das } \\
\text { Ações de Vigilância }\end{array}$ & Busca ativa $(10,0)$ & $\begin{array}{l}\text { Busca de faltosos ao tratamento de } \\
\text { tuberculose }\end{array}$ & 2,5 & 2,5 & * \\
\hline \multirow[t]{11}{*}{ Epidemiológica } & & $\begin{array}{l}\text { Busca de faltosos ao tratamento de } \\
\text { hanseníase }\end{array}$ & 2,5 & 2,5 & * \\
\hline & & Busca de comunicantes de tuberculose & 2,5 & 2,5 & * \\
\hline & & Busca de comunicantes de hanseníase & 2,5 & 2,5 & * \\
\hline & Investigação $(10,0)$ & $\begin{array}{l}\text { Investigação dos casos de doenças } \\
\text { exantemáticas }\end{array}$ & 2,0 & 2,0 & * \\
\hline & & Investigação dos casos de dengue & 2,0 & 2,0 & * \\
\hline & & Investigação de óbitos infantis & 2,0 & 2,0 & * \\
\hline & & Investigação de óbitos maternos & 2,0 & 2,0 & 5,0 \\
\hline & & Existência de fichas de investigação & 2,0 & 2,0 & 5,0 \\
\hline & $\begin{array}{l}\text { Educação em saúde } \\
(10,0)\end{array}$ & $\begin{array}{l}\text { Realização de atividades educativas } \\
\text { abordando agravos de interesse da } \\
\text { vigilância epidemiológica }\end{array}$ & 3,34 & 3,34 & 3,34 \\
\hline & & $\begin{array}{l}\text { Tipo das atividades educativas (contínua } \\
\text { e sistemática) }\end{array}$ & 3,33 & 3,33 & 3,33 \\
\hline & & $\begin{array}{l}\text { Existência de folders, cartazes e material } \\
\text { educativo dos principais agravos }\end{array}$ & 3,33 & 3,33 & 3,33 \\
\hline
\end{tabular}

PPI-VS: Programação Pactuada e Integrada; PSF: Programa Saúde da Família; SIM: Sistema de Informações sobre Mortalidade; SINASC: Sistema de Informações de Nascidos Vivos; SINAN: Sistema de Informação de Agravos de Notificação; SIS: Sistema Integrado de Saúde.

* Nível de análise não abordado com critério em questão.

\section{Resultados}

A dimensão unitária da vigilância epidemiológica no Recife foi classificada como parcialmente implantada, enquanto os componentes Gestão da Vigilância Epidemiológica e Desenvolvimento das Ações da Vigilância Epidemiológica foram classificados como parcialmente implantados $\mathrm{e}$ implantados, respectivamente. Alguns critérios considerados na avaliação foram incipientes nos três níveis do sistema municipal de vigilância epidemiológica, como a Normatização das ações e as atividades de Monitoramento e Avaliação (Tabela 2).

A vigilância epidemiológica do nível central foi classificada como implantada, mesmo havendo dificuldades em atender a alguns dos critérios normatizados. Na Gestão da Vigilância Epidemiológica, a existência e a elaboração de normas internas definindo as atribuições para cada nível do sistema (Normatização) foram critérios não observados, mas não interferiram na classificação do grau de implantação; do componente considerado implantado. No Desenvolvi- mento das Ações de Vigilância Epidemiológica, os dois subcomponentes avaliados, Investigação e Educação em Saúde, apresentaram todas as atividades realizadas, classificando a vigilância epidemiológica neste componente como implanta$d a$ (Tabela 2).

Nos distritos sanitários, a vigilância epidemiológica foi considerada parcialmente implantada. Na análise por componentes do modelo lógico, a Gestão da Vigilância Epidemiológica foi classificada como parcialmente implantada, devido, principalmente, à insuficiência de Recursos Humanos e à incipiente realização de atividades de Planejamento e Monitoramento/Avaliação. O componente Desenvolvimento das Ações de Vigilância Epidemiológica foi classificado como implantado, ainda que as atividades relacionadas à Busca Ativa e à Notificação tenham obtido uma baixa pontuação com relação aos outros subcomponentes (Tabela 2).

A vigilância epidemiológica das equipes de saúde da família foi classificada como parcialmente implantada. A insuficiente adesão às normas no componente Gestão da Vigilância Epi- 
Escores e grau de implantação segundo níveis, componentes, subcomponentes e dimensão unitária da vigilância epidemiológica. Recife, Pernambuco, Brasil, 2005.

\begin{tabular}{|c|c|c|c|c|c|c|}
\hline \multirow[t]{2}{*}{ Componente } & \multirow[t]{2}{*}{ Subcomponente } & \multirow[t]{2}{*}{ Critérios } & \multicolumn{4}{|c|}{ Pontuação obtida } \\
\hline & & & $\begin{array}{l}\text { Equipes de } \\
\text { saúde da } \\
\text { família }\end{array}$ & $\begin{array}{l}\text { Distritos } \\
\text { sanitários }\end{array}$ & $\begin{array}{l}\text { Nível } \\
\text { central }\end{array}$ & Recife \\
\hline \multirow{32}{*}{$\begin{array}{l}\text { Gestão da } \\
\text { Vigilância } \\
\text { Epidemiológica }\end{array}$} & Recursos & Diretor(a) da vigilância epidemiológica & * & 2,77 & 3,34 & 8,33 ** \\
\hline & humanos $(10,0)$ & nomeado com carga horária mínima de & & & & \\
\hline & & 40 horas & & & & \\
\hline & & Profissionais de nível superior com carga & * & 0,56 & 3,33 & \\
\hline & & horária mínima de 20 horas & & & & \\
\hline & & Profissionais de nível médio com carga & * & 1,66 & 3,33 & \\
\hline & & horária mínima de 20 horas & & & & \\
\hline & & $\begin{array}{l}\text { Profissionais das equipes da saúde da } \\
\text { família que realizam ações de vigilância } \\
\text { epidemiológica }\end{array}$ & 10,0 & * & * & \\
\hline & & Subtotal & 10,0 ** & 5,0 & 10,0 ** & \\
\hline & Planejamento das & Planejamento das ações & 4,08 & 3,33 & 5,0 & 8,0 ** \\
\hline & ações $(10,0)$ & $\begin{array}{l}\text { Acompanhamento da programação das } \\
\text { ações e metas }\end{array}$ & 4,08 & 2,5 & 5,0 & \\
\hline & & Subtotal & 8,16 ** & $5,83 * \star \star$ & 10,0 ** & \\
\hline & $\begin{array}{l}\text { Monitoramento e } \\
\text { avaliação }(10,0)\end{array}$ & $\begin{array}{l}\text { Construção de gráfico de tendência dos } \\
\text { agravos }\end{array}$ & * & 0,37 & 1,25 & $6,03 \#$ \\
\hline & & Monitoramento dos óbitos infantis & * & 0,93 & 1,25 & \\
\hline & & Monitoramento dos óbitos maternos & * & 0,74 & 1,25 & \\
\hline & & Monitoramento dos casos de tuberculose & 1,6 & * & * & \\
\hline & & Monitoramento dos casos de hanseníase & 16 & * & * & \\
\hline & & notificados nas unidades de referência & & & & \\
\hline & & Recebimento de boletins de & * & 1,11 & * & \\
\hline & & acompanhamento de tuberculose e & & & & \\
\hline & & hanseníase & & & & \\
\hline & & Monitoramento das metas da PPI-VS & 0,36 & 0,56 & 1,25 & \\
\hline & & $\begin{array}{l}\text { Monitoramento dos indicadores do Pacto } \\
\text { da Atenção Básica }\end{array}$ & 0,48 & 0 & 0 & \\
\hline & & $\begin{array}{l}\text { Reuniões com os técnicos do distrito } \\
\text { sanitário para avaliação das ações }\end{array}$ & * & 0,37 & 1,25 & \\
\hline & & $\begin{array}{l}\text { Reuniões com os profissionais do PSF para } \\
\text { avaliação das ações }\end{array}$ & 1,04 & 0,19 & 1,25 & \\
\hline & & $\begin{array}{l}\text { Construção de instrumento para avaliação } \\
\text { das ações de vigilância epidemiológica }\end{array}$ & * & 0 & 1,25 & \\
\hline & & Subtotal & 5,08 & 4,26 & $8,75 * *$ & \\
\hline & Integração das & Ações realizadas em conjunto com as & * & 5,0 & 5,0 & 9,0 ** \\
\hline & ações $(10,0)$ & Vigilâncias Sanitária e Ambiental & & & & \\
\hline & & Ações realizadas em conjunto com a & * & 3,0 & 5,0 & \\
\hline & & Atenção Básica & & & & \\
\hline & & Subtotal & * & 8,0 ** & 10,0 ** & \\
\hline
\end{tabular}

(continua) 
Tabela 2 (continuação)

\begin{tabular}{|c|c|c|c|c|c|c|}
\hline \multirow[t]{2}{*}{ Componente } & \multirow[t]{2}{*}{ Subcomponente } & \multirow[t]{2}{*}{ Critérios } & \multicolumn{4}{|c|}{ Pontuação obtida } \\
\hline & & & $\begin{array}{l}\text { Equipes de } \\
\text { saúde da } \\
\text { família }\end{array}$ & $\begin{array}{l}\text { Distritos } \\
\text { sanitários }\end{array}$ & $\begin{array}{c}\text { Nível } \\
\text { central }\end{array}$ & Recife \\
\hline Gestão da & Informação $(10,0)$ & Processamento e análise dos dados no SIM & * & * & 1,0 & $7,83 \#$ \\
\hline Vigilância & & Processamento e análise dos dados no & * & * & 1,0 & \\
\hline \multirow[t]{21}{*}{ Epidemiológica } & & SINASC & & & & \\
\hline & & Processamento e análise dos dados no & * & 1,43 & * & \\
\hline & & SINAN & & & & \\
\hline & & $\begin{array}{l}\text { Envio do banco de dados do SIM, SINASC } \\
\text { e SINAN para o estado }\end{array}$ & * & * & 1,0 & \\
\hline & & $\begin{array}{l}\text { Envio de boletins epidemiológicos aos } \\
\text { outros níveis }\end{array}$ & * & 0,48 & 1,0 & \\
\hline & & $\begin{array}{l}\text { Envio dos bancos de dados dos SIS aos } \\
\text { níveis normatizados }\end{array}$ & 1,9 & 1,43 & 1,0 & \\
\hline & & $\begin{array}{l}\text { Consolidação e análise dos dados de } \\
\text { vigilância epidemiológica produzidos pelas } \\
\text { equipes de saúde da família }\end{array}$ & 1,88 & * & * & \\
\hline & & $\begin{array}{l}\text { Produção de relatórios com a situação } \\
\text { epidemiológica da área }\end{array}$ & 0,73 & * & 1,0 & \\
\hline & & Existência de computadores & 0,65 & 1,19 & 1,0 & \\
\hline & & Existência de impressoras & * & 1,43 & 1,0 & \\
\hline & & Existência de telefones exclusivos & * & 1,19 & 1,0 & \\
\hline & & $\begin{array}{l}\text { Suprimento de papel, tinta de impressora, } \\
\text { disquete }\end{array}$ & * & 1,19 & 1,0 & \\
\hline & & Subtotal & $5,16 * \star \star$ & $8,33 * \star$ & $10,0 * \star$ & \\
\hline & $\begin{array}{l}\text { Normatização } \\
(10,0)\end{array}$ & $\begin{array}{l}\text { Elaboração de normas internas definindo } \\
\text { atribuições de cada nível do sistema }\end{array}$ & * & * & 0 & 5,58 *** \\
\hline & & $\begin{array}{l}\text { Utilização das normas existentes para } \\
\text { realização das ações de vigilância } \\
\text { epidemiológica }\end{array}$ & 1,88 & 3,33 & 2,5 & \\
\hline & & Existência de Leis/Portarias; guias do & 1,93 & 3,33 & 2,5 & \\
\hline & & Ministério da Saúde & & & & \\
\hline & & $\begin{array}{l}\text { Existência de normatização interna sobre } \\
\text { as atribuições de cada nível do sistema }\end{array}$ & 1,23 & 0 & 0 & \\
\hline & & Subtotal & 5,04 *** & $6,66 \#$ & $5,0 \star \star \star$ & \\
\hline & & Grau de implantação da Gestão da & $6,69 \#$ & $6,35 \#$ & 8,96 & $7,33 \#$ \\
\hline & & Vigilância Epidemiológica & & & & \\
\hline $\begin{array}{l}\text { Desenvolvimento } \\
\text { das Ações }\end{array}$ & Notificação $(10,0)$ & $\begin{array}{l}\text { Notificação dos casos suspeitos de } \\
\text { doenças exantemáticas }\end{array}$ & 1,67 & 2,0 & * & $7,62 \#$ \\
\hline de Vigilância & & Notificação negativa de sarampo & 0,69 & * & * & \\
\hline Epidemiológica & & Notificação de casos suspeitos de dengue & 1,45 & 2,0 & * & \\
\hline & & Notificação de casos de tuberculose & 1,67 & 0,67 & * & \\
\hline & & Notificação de casos de hanseníase & 1,61 & 0 & * & \\
\hline & & Existência de fichas de notificação & 1,48 & 2,0 & * & \\
\hline & & Subtotal & $8,57 \star \star$ & $6,67 \#$ & * & \\
\hline
\end{tabular}

(continua) 
Tabela 2 (continuação)

\begin{tabular}{|c|c|c|c|c|c|c|}
\hline \multirow[t]{2}{*}{ Componente } & \multirow[t]{2}{*}{ Subcomponente } & \multirow[t]{2}{*}{ Critérios } & \multicolumn{4}{|c|}{ Pontuação obtida } \\
\hline & & & $\begin{array}{l}\text { Equipes de } \\
\text { saúde da } \\
\text { família }\end{array}$ & $\begin{array}{l}\text { Distritos } \\
\text { sanitários }\end{array}$ & $\begin{array}{l}\text { Nível } \\
\text { central }\end{array}$ & Recife \\
\hline $\begin{array}{l}\text { Desenvolvimento } \\
\text { das Ações }\end{array}$ & Diagnóstico $(10,0)$ & $\begin{array}{l}\text { Sorologia para diagnóstico de casos de } \\
\text { sarampo }\end{array}$ & 1,66 & 5,0 & * & 8,97 ** \\
\hline \multirow[t]{20}{*}{$\begin{array}{l}\text { de Vigilância } \\
\text { Epidemiológica }\end{array}$} & & $\begin{array}{l}\text { Sorologia para diagnóstico de casos de } \\
\text { denque }\end{array}$ & 1,85 & 5,0 & * & \\
\hline & & $\begin{array}{l}\text { Exame clínico e baciloscópico de suspeitos } \\
\text { de hanseníase }\end{array}$ & 2,05 & * & * & \\
\hline & & $\begin{array}{l}\text { Baciloscopia direta do escarro de casos } \\
\text { suspeitos de tuberculose }\end{array}$ & 2,38 & * & * & \\
\hline & & Subtotal & 7,94 \# & 10,0 ** & * & \\
\hline & Busca ativa $(10,0)$ & $\begin{array}{l}\text { Busca de faltosos ao tratamento de } \\
\text { tuberculose }\end{array}$ & 2,5 & 0,42 & * & $6,25 \#$ \\
\hline & & $\begin{array}{l}\text { Busca de faltosos ao tratamento de } \\
\text { hanseníase }\end{array}$ & 2,5 & 0,42 & * & \\
\hline & & Busca de comunicantes de tuberculose & 2,5 & 0,83 & * & \\
\hline & & Busca de comunicantes de hanseníase & 2,5 & 0,83 & * & \\
\hline & & Subtotal & 10,0 ** & 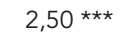 & * & \\
\hline & Investigação $(10,0)$ & $\begin{array}{l}\text { Investigação dos casos de doenças } \\
\text { exantemáticas }\end{array}$ & 1,52 & 2,0 & * & 8,92 ** \\
\hline & & Investigação dos casos de dengue & 1,19 & 2,0 & & \\
\hline & & Investigação de óbitos infantis & 1,77 & 2,0 & * & \\
\hline & & Investigação de óbitos maternos & 1,35 & 1,0 & 5,0 & \\
\hline & & Existência de fichas de investigação & 1,93 & 2,0 & 5,0 & \\
\hline & & Subtotal & $7,76 \#$ & 9,0 ** & 10,0 ** & \\
\hline & $\begin{array}{l}\text { Educação em } \\
\text { saúde }(10,0)\end{array}$ & $\begin{array}{l}\text { Realização de atividades educativas } \\
\text { abordando agravos de interesse da } \\
\text { vigilância epidemiológica }\end{array}$ & 3,0 & 3,33 & 3,34 & 8,67 ** \\
\hline & & $\begin{array}{l}\text { Tipo das atividades educativas (contínua e } \\
\text { sistemática) }\end{array}$ & 2,26 & 3,33 & 3,33 & \\
\hline & & $\begin{array}{l}\text { Existência de folders, cartazes e material } \\
\text { educativo dos principais agravos }\end{array}$ & 2,42 & 1,67 & 3,33 & \\
\hline & & Subtotal & $7,68 \#$ & 8,33 & 10,0 & \\
\hline & & $\begin{array}{l}\text { Grau de implantação da dimensão unitária } \\
\text { da vigilância epidemiológica }\end{array}$ & 8,39 & 7,30 \# & 10,0 & 8,56 ** \\
\hline \multicolumn{3}{|c|}{ Grau de implantação da dimensão unitária da vigilância epidemiológica } & 7,54 \# & $6,82 \#$ & 9,48 & 7,95 \# \\
\hline
\end{tabular}

PPI-VS: Programação Pactuada e Integrada; PSF: Programa Saúde da Família; SIM: Sistema de Informações sobre Mortalidade; SINASC: Sistema de Informações de Nascidos Vivos; SINAN: Sistema de Informação de Agravos de Notificação; SIS: Sistema Integrado de Saúde.

Grau de implantação:

* Nível de análise não abordado com critério em questão;

** Implantada;

*** Não implantada;

\# Parcialmente implantada. 
demiológica esteve relacionada ao incipiente monitoramento das metas da PPI-VS e do Pacto de indicadores da Atenção Básica (Monitoramento/Avaliação) e à inexistência de normas definindo as atribuições de cada nível do sistema (Normatização), o que contribuiu para sua classificação como parcialmente implantada. No componente Desenvolvimento das Ações de Vigilância Epidemiológica, a maior parte das equipes de saúde da família não realiza investigação dos casos de dengue e dos óbitos maternos (Investigação), e não faz atividades educativas de forma contínua e sistemática (Educação em Saúde), embora este componente tenha obtido a classificação implantada (Tabela 2).

\section{Discussão}

Os critérios utilizados para a avaliação da implantação da vigilância epidemiológica no Município do Recife possibilitaram classificá-la como parcialmente implantada, sendo que dos três níveis analisados, em dois - distritos sanitários e equipes de saúde da família - a vigilância epidemiológica esteve parcialmente implantada, e apenas no nível central esteve implantada. Embora os pontos de corte utilizados tenham reconstruído a realidade empiricamente esperada, os instrumentos não foram capazes de assegurar o que, de fato, deve ocorrer em cada nível.

A estratégia metodológica utilizada neste estudo permitiu abordar as principais questões para avaliação da vigilância epidemiológica num nível municipal, considerando a transversalidade do tema no SUS. Vários autores 9,11,16,17,18 têm utilizado estratégias similares, no intuito de apreender o objeto estudado o mais próximo de sua realidade. Todavia, a coerência entre o modelo lógico elaborado e os achados empíricos não foi suficiente para detalhar as atribuições dos níveis de análise, no que se refere às atividades de vigilância epidemiológica. A insuficiente definição quanto à organização e atribuições da vigilância epidemiológica em sistemas municipais de saúde, considerando os diversos níveis de gestão e desenvolvimento das atividades (central, distrital e unidades de saúde) dificultaram a avaliação da dimensão normativa. Essa constatação sinaliza a dinamicidade e transitoriedade do modelo lógico adotado, que na medida em que incorpora definições e normas necessita se aperfeiçoar.

Os critérios avaliados no subcomponente Normatização traduzem essa realidade. Os três níveis possuem normas ministeriais, embora apenas metade das equipes de saúde da família as utilize; todavia, não possuem normas específicas definindo atribuições dos níveis do sistema municipal, o que pode ter resultado na sobreposição ou na omissão de algumas atividades. $\mathrm{Ou}$ seja, o sistema municipal não possuindo regras explícitas quanto ao funcionamento da vigilância epidemiológica, dificulta o entendimento dos profissionais sobre as especificidades ou complementaridades das ações em cada nível 8, fato determinante no grau de implantação obtido.

Devido à pouca clareza das normas existentes, no nível central, a realização de atividades de apenas dois subcomponentes, Investigação e Ações Educativas, classificou o Desenvolvimento das Ações de Vigilância Epidemiológica como implantado, com uma pontuação maior do que a Gestão de Vigilância Epidemiológica, que é, a priori, seu componente essencial. Esse achado sugere reflexões antagônicas, porém plausíveis. A primeira, que esforços seriam minimizados se essas atividades fossem executadas por níveis mais apropriados dos conhecimentos da área adscrita (este nível deveria realizar este tipo de atividade?). A segunda, que as vantagens estruturais (equipamentos, recursos humanos) do nível central, quando comparadas aos demais, propiciam a centralização dessas atividades (a descentralização das ações de vigilância epidemiológica, neste caso, é viável?).

A insuficiência de recursos humanos (técnicos de níveis superior e médio) na vigilância epidemiológica dos distritos sanitários, que são a instância intermediária - suporte tanto do nível central quanto das equipes de saúde da família - reforça a segunda reflexão. Os documentos analisados agregam o maior número de atividades nesse nível, quando na prática, atividades relacionadas à informação, diagnóstico, investigação e educação em saúde são priorizadas, em detrimento das atividades de planejamento, monitoramento/avaliação e busca ativa. A relação direta entre a carência de recursos humanos e a dificuldade de descentralização das ações de vigilância epidemiológica para os níveis do sistema foi relatada por Goldbaum 19 e Passos ${ }^{8}$. Para Villa et al. 20, a descentralização das ações de vigilância epidemiológica para os níveis distritais e para as unidades básicas de saúde só se torna presumível à proporção que se ofereçam profissionais qualificados e infra-estrutura compatível com suas atribuições.

Nas equipes de saúde da família, embora todos os profissionais das equipes realizem, de alguma forma, ações de vigilância epidemiológica, o monitoramento dos indicadores da área e a consolidação/análise da informação gerada pela equipe - atividades relacionadas à Gestão da Vigilância Epidemiológica - ainda são atividades pouco realizadas. $\mathrm{O}$ foco da intervenção das equipes de saúde da família são as atividades de busca ativa, notificação e diagnóstico, diferen- 
te do que foi verificado por Santos \& Melo $21 \mathrm{e}$ por Cerqueira et al. ${ }^{22}$, que embora a descentralização das ações de vigilância epidemiológica para o Programa Saúde da Família (PSF) tenha contribuído para a melhoria de alguns indicadores de saúde, os profissionais ainda consideram uma prática secundária no processo de trabalho, que deveria ser realizada por profissionais especializados.

Em que pese a descentralização das ações de vigilância epidemiológica para os municípios, observa-se diferenciais importantes quanto à adesão ao preconizado entre os diversos níveis organizacionais da vigilância epidemiológica no sistema municipal de saúde. Essa constatação aponta necessidades diferenciadas para garantir o desenvolvimento de ações conforme as recomendações das instâncias gestoras do sistema nacional de saúde.

Os distritos sanitários precisam agregar recursos humanos aos setores de vigilância epidemiológica para desenvolver todas as atividades normatizadas; as equipes de saúde da família reconhecer o monitoramento dos indicadores de saúde da área e a informação produzida como atividades importantes para redirecionar as práticas. Entretanto, a elaboração de normas específicas definindo as atribuições dos três níveis, atividade a ser desencadeada pelo nível central, seria anterior e determinante nas demais.

Embora este estudo tenha apontado algumas fragilidades e potencialidades da vigilância epidemiológica municipal em distintos níveis do sistema, o modelo lógico e a matriz de indicadores utilizados não devem ser considerados instrumentos definitivos, pela complexidade do objeto avaliado e pelo estágio de desenvolvimento da descentralização da ação na instância municipal. Além disso, as condições traçadoras e os eventos sentinela selecionados podem não dar conta das especificidades de cada realidade.

Nesse sentido, algumas recomendações poderiam ser feitas para o aprimoramento da vigilância epidemiológica municipal:

- A definição de atribuições dos níveis do sistema de vigilância epidemiológica municipal, evitando a sobreposição ou omissão de algumas atividades;

- O aperfeiçoamento dos instrumentos utilizados, no sentido de captar mais fidedignamente as nuances de cada nível;

- A realização de avaliações mais aprofundadas que expliquem os resultados encontrados.

Para subsidiar o movimento de repensar a política de descentralização da vigilância epidemiológica, agora no espaço intramunicipal, o efetivo envolvimento de todos os atores responsáveis pelas ações, desde os representantes políticos, profissionais de saúde até os líderes comunitários torna-se imprescindível. A participação de todos esses grupos permite a incorporação de visões diferentes no processo da avaliação, e assegura a imparcialidade das informações, além de aumentar a relevância e a credibilidade dos resultados obtidos 21 .

\section{Resumo}

O objetivo deste estudo foi avaliar o grau de implantação da vigilância epidemiológica na Secretaria Municipal de Saúde do Recife, Pernambuco, Brasil. A estratégia do trabalho consistiu num estudo de caso utilizando-se uma abordagem normativa. Foi delineado o modelo lógico da vigilância epidemiológica, a partir do qual foram selecionados critérios que compuseram uma matriz submetida a especialistas usando-se a técnica de consenso - método Delphos. Essa matriz consensuada originou os questionários das entrevistas. Foram abordados três níveis de análise: nível central, 6 distritos sanitários e 62 equipes de saúde da família. O estudo revelou que a vigilância epidemiológica está parcialmente implantada no município, a partir do grau de implantação dos três níveis de análise, sendo o nível central o único em que a vigilância epidemiológica encontra-se implantada, enquanto que nos distritos sanitários e nas equipes de saúde da família está parcialmente implantada. Em que pese a descentralização das ações de vigilância epidemiológica para os municípios, os níveis do sistema municipal possuem necessidades diferenciadas para atender ao que está normatizado, entre elas, a elaboração de normas internas especificando as atribuições dos três níveis.

Avaliação em Saúde; Saúde da Família; Vigilância Epidemiológica 


\section{Colaboradores}

L. C. A. Bezerra, E. Freese, P. G. Frias participaram igualmente de todas as etapas da elaboração do artigo. I. Samico participou da técnica de consenso e da redação do texto final. C. K. A. Almeida participou da revisão bibliográfica e da redação do texto final.

\section{Referências}

1. Coordenação-Geral e Apoio à Gestão Descentralizada, Departamento de Apoio à Descentralização, Secretaria Executiva, Ministério da Saúde. Diretrizes operacionais dos pactos pela vida, em defesa do SUS e de gestão. Brasília: Ministério da Saúde; 2006. (Série A. Normas e Manuais Técnicos).

2. Centro de Educação e Assessoramento Popular. Pacto pela saúde: possibilidade ou realidade? Passo Fundo: Instituto Superior de Filosofia Berthier; 2007

3. Ribeiro P. Pacto pela saúde 2006: o SUS não muda. O que muda no SUS? http://www.ensp.fiocruz.br/ eventos_novo/dados/arq2750.pdf (acessado em $10 / \mathrm{jul} / 2008$ )

4. Fundação Nacional de Saúde. Guia de vigilância epidemiológica. Brasília: Ministério da Saúde; 2005.

5. Ministério da Saúde. Programação Pactuada e Integrada 2006. http://portal.saude.gov.br/portal/ arquivos/pdf/ppi_vs_2006_regioes_nordeste2.pdf (acessado em 10/Abr/2008).

6. Fundação Nacional de Saúde. Projeto VIGISUS: diretrizes e procedimentos para o apoio de estudos e pesquisas no âmbito do VIGISUS. Componente II. Brasília: Ministério da Saúde; 2004.

7. Carvalho MS, Marzocchi KBF. Avaliação da prática de vigilância epidemiológica nos serviços públicos de saúde no Brasil. Rev Saúde Pública 1992; 26: 66-74.

8. Passos IMR. "Assistir" e "vigiar" as ações de vigilância epidemiológica nas unidades básica de saúde. Avanços e perspectivas [Tese de Doutorado]. Ribeirão Preto: Escola de Enfermagem de Ribeirão Preto; 2003.
9. Pimenta Júnior FG. Desenvolvimento e validação de um instrumento para avaliar o Programa Nacional de Controle da Dengue no âmbito municipal [Dissertação de Mestrado]. Rio de Janeiro: Escola Nacional de Saúde Pública, Fundação Oswaldo Cruz; 2005.

10. Silva Júnior JB. Epidemiologia em serviço: uma avaliação de desempenho do Sistema Nacional de Vigilância em Saúde [Tese de Doutorado]. Campinas: Faculdade de Ciências Médicas, Universidade Estadual de Campinas; 2004.

11. Souza LEPF, Silva LMV, Hartz Z, Paim JS. Conferência de consenso sobre a imagem-objetivo da descentralização da atenção à saúde no Brasil. In: Hartz ZMA, Vieira-da-Silva LM, organizadores. Avaliação em saúde: dos modelos teóricos à prática na avaliação de programas e sistemas de saúde. Salvador: Edufba/Rio de Janeiro: Editora Fiocruz; 2005. p. 41-74.

12. Kessner M, Kalk CE, Singer J. Evaluación de la calidad de la salud por el método de los procesos trazadores. In: White KL, editor. Investigaciones sobre servicios de salud: una antología. Washington DC: Organización Panamericana de la Salud; 1992. p. 555-63. (Publicación Científica, 534).

13. Rutstein DD. Measuring the quality of medical care: a clinical method. N Engl J Med 1976; 294: 582-8.

14. Felisberto E, Carvalho EF, Maggi RS, Samico I. Avaliação do processo de implantação da estratégia da Atenção Integrada às Doenças Prevalentes da Infância no Programa Saúde da Família, no Estado de Pernambuco, Brasil. Cad Saúde Pública 2002; 18:1737-45. 
15. Samico I, Hartz ZMA, Felisberto E, Carvalho EF. Atenção à saúde da criança: uma análise do grau de implantação e da satisfação de profissionais e usuários em dois municípios do Estado de Pernambuco, Brasil. Rev Bras Saúde Matern Infant 2005; 5:229-40.

16. Saraceni V, Vellozo VRO, Leal MC, Hartz ZMA. Avaliação das campanhas para a eliminação da sífilis congênita, no Município do Rio de Janeiro, a partir de um modelo teórico lógico. Rev Bras Saúde Matern Infant 2005; 5 Suppl 1:S33-41.

17. Oliveira MIC, Camacho LAB, Souza IEO. Promoção, proteção e apoio à amamentação na atenção primária à saúde no Estado do Rio de Janeiro, Brasil: uma política de saúde pública baseada em evidência. Cad Saúde Pública 2005; 21:1901-10.

18. Hartz ZMA, Champagne F, Contandriopoulos AP, Leal MC. Avaliação do programa materno-infantil: análise de implantação em sistemas locais de saúde no nordeste do Brasil. In: Hartz ZMA, organizadora. Avaliação em saúde: dos modelos conceituais à prática na análise da implantação de programas. Rio de Janeiro: Editora Fiocruz; 1997. p. 89-131.
19. Goldbaum M. Epidemiologia e serviços de saúde. Cad Saúde Pública 1996; 12 Suppl 2:95-8.

20. Villa TCS, Palha PF, Muniz JN, Cardozo-Gonzales RI, Pinto Neto JM, Assis MMA. A vigilância epidemiológica e a perspectiva de trabalho no território - Secretaria Municipal de Saúde - Ribeirão Preto. Rev Latinoam Enferm 2002; 10:21-7.

21. Santos SSBS, Melo CMM. Avaliação da descentralização da vigilância epidemiológica para a equipe de saúde da família. Ciênc Saúde Coletiva 2006; 13:1923-32.

22. Cerqueira EM, Assis MMA, Villa TCS, Leite JA. Vigilância Epidemiológica no processo de municipalização do Sistema de Saúde de Feira de Santana - BA. Epidemiol Serv Saúde 2003; 12:213-23.

Recebido em 23/Jul/2008

Versão final reapresentada em 15/Out/2008 Aprovado em 24/Out/2008 